\title{
Hardware Determination for a Spherical Attitude Control Prototype
}

\author{
John T. Compas \\ Severson Group in the WEMPEC Lab \\ Computer Engineering, University of Wisconsin- \\ Madison
}

\begin{abstract}
To find alternative approaches to conventional reaction wheels, a spherical reaction device utilizing magnetic bearings is proposed. A single spherical rotor could provide three-axis control and thereby solve many of the issues with conventionally lubricated reaction wheels. To determine and gain experience with the hardware needed to create such a prototype, a simpler one-degree-of-freedom design is described in this paper. The reduced complexity of this system allows many different topologies to be tested quickly while still retaining enough intricacy to be useful. After a few attempts at levitation with an analog control circuit and other microcontrollers, the Advanced Motor Drive Controller was utilized. Although still under development in the Severson Group at the Wisconsin Electric Machines and Power Electronics (WEMPEC) lab, the AMDC will provide enough control capabilities to eventually manage the one DOF test case and then, ultimately, final prototype.
\end{abstract}

\section{Introduction}

The motivation for this research stems from issues with a common method of spacecraft attitude control called reaction wheels. They usually consist of four wheels in a tetrahedral orientation, and angular momentum is exchanged between them and the spacecraft to rotate it. Multiple satellites have suffered from reaction wheel failure due to lubrication issues in these wheels (Carrara \& Kuga, 2015). These relatively inexpensive components can derail or shorten high value missions. Conventional reaction wheels utilizing magnetic bearings are prohibitively expensive and have found use in relatively fewer missions (C.U.Mba, et al., 2017).

Spherical rotors that rotate independently of the craft's current attitude, can provide three axis control with one rotor. Fewer rotors could offset the additional weight of electromagnets and control systems needed by magnetic bearings. This simplicity could additionally benefit microsatellites traveling outside of Earth's magnetic field where other attitude systems like magnetorquers could not be used as they require an external magnetic field to operate.

Another group has tested reaction devices like the one proposed here and has encountered issues with the efficiency of the system. The torque per unit power outputted was too low to make the system feasible for satellites (Iwakura, Tsuda, \& Tsuda, 2008). The efficiency of this system could potentially be increased by using hollow spheres or other methods, but a solid sphere with six electromagnets is the primary goal.

Before this full prototype is realized, proper measurement techniques and the control systems need to be developed and tested. A simpler one degree of freedom system was devised, consisting of a sphere being levitated beneath a single electromagnet. Multiple designs were tested and failed, starting with a purely analog control system with operational amplifiers to digital systems utilizing a TI microcontroller. Eventually, a Xilinx Zynq-7000 processor, first on

Funding for this research was provided by the Wisconsin Space Grant Consortium 
a Zedboard, and then on the Advanced Motor Drive Controller (AMDC) was used to control the system. The AMDC is a custom carrier card for the PicoZed; it was designed by the Severson group within the WEMPEC lab. The PicoZed is a system-on-module (SOM) chip that contains a Zynq-7000 processor and peripherals to utilize it. Since the board is still under development, a significant amount of time was spent refining and solving issues with the board. However, when operational, the AMDC will have significant control and measurement capabilities which will enable it to be used throughout the development of the full 3D prototype.

\section{Materials and Methods}

As shown in "Design of a Magnetic Levitation Control System; An Undergraduate Project" (Wong, 1986) and "Electromagnetic Design of a Magnetic Suspension System" (Hurley \& Wolfle, 1997), levitating a sphere electromagnetically is an exercise in many undergraduate classes and studies. As such, the control parameters of the system are well described and known. "Electromagnetic Design of a Magnetic Suspension System" was especially useful in providing optimizations to the electromagnet design. A sphere one inch in diameter was chosen because of the low power requirements to levitate it, its similarity in size to other systems, and the wide availability of spheres this size. The theoretical control of the system is first derived, and then applied to the more detailed hardware implementation of the control system.

\subsection{Theoretical Control System}

The theoretical control of the system in one dimension is first analyzed. The coordinates in this system are set so that the origin of this system is at the mid-point of the bottom of the electromagnet core. The $Z$ axis is parallel to the force due to gravity and increasingly positive away from the ground, as shown in Fig 1. There is no significant force in the $X Y$ plane, so control in those directions is not analyzed. At equilibrium, the force due to gravity will be equal to the force of the electromagnet. The force from the electromagnet should be inversely proportional to the square of the distance as shown in Wong, 1986. A beam formed by an IR LED and a phototransistor is placed across the equilibrium point to sense the position of the sphere near it. At equilibrium, this system has the form:

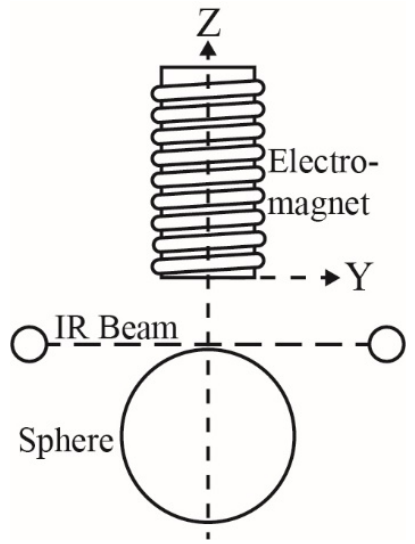

Figure 1: Cross section of System Design Diagram

$$
M_{s} g=M_{s} \frac{\partial^{2} z}{\partial t^{2}}=f(z, i)=C \frac{i^{2}}{z^{2}}
$$

$M_{s}=$ Mass of Sphere

$g=$ Acceleration due to Gravity

$i=$ Electromagnet current

The constant $C$ is determined by many factors and will be estimated later in simulation. As a non-linear system, simple control techniques cannot be applied, but the system can be linearized around the equilibrium point, $f\left(z_{s}, i_{s}\right)$ as shown below. Small perturbations around this point will be denoted $\Delta z$ and $\Delta i$. Taking the Taylor expansion of $f$ and ignoring all second and greater order terms yields the following: 


$$
f(z, i) \approx f\left(z_{s}, i_{s}\right)+\left.\frac{\partial f}{\partial z}\right|_{z_{s}, i_{s}} \Delta z+\left.\frac{\partial f}{\partial i}\right|_{z_{s}, i_{s}} \Delta i=f\left(z_{s}, i_{s}\right)-\frac{2 C i_{s}^{2}}{z_{S}^{3}} \Delta z+\frac{2 C i_{s}}{z_{S}^{2}} \Delta i
$$

Letting constants $C_{z}=\frac{2 C i_{s}^{2}}{z_{s}^{3}}$ and $C_{i}=\frac{2 C i_{s}}{z_{s}^{2}}$ and then substituting (2) into (1) gives:

$$
\frac{\partial \Delta z^{2}}{\partial t^{2}}=\frac{\left(-C_{z} \Delta z+C_{i} \Delta i\right)}{M_{S}}
$$

Taking the Laplace transform of the above yields the plant transfer function, $G_{p}(S)$. $S$ represents both a real, $\sigma$, and complex, $\omega$, component so $S=\sigma+\omega j$. The use of $S$ in the Laplace domain facilitates the representation and manipulation of differential equations.

$$
G_{p}(S)=\frac{\Delta Z(S)}{\Delta I(S)}=\frac{C_{i}}{M_{S} S^{2}-C_{z}}=\frac{\frac{C_{i}}{M_{S}}}{S^{2}-\frac{C_{z}}{M_{S}}}
$$

As (4) is an unstable system with two poles at $\pm \sqrt{\frac{C_{z}}{M_{s}}}$, active control is needed to stabilize the system. A proportional-derivative (PD) controller is chosen because of its simplicity, well described characteristics, and use in similar systems. The transfer function of the controller, $G_{P D}(S)$ has two constants $K_{p}$ and $K_{d}$. The values of these can be chosen to stabilize the system as needed. This gives the full transfer functions:

$$
G_{P D}(S)=K_{p}+K_{d} S
$$

The phototransistor sensor beam is situated so that the center of the beam is at the equilibrium point, or set point (S.P.) to maximize its effective range. As zero voltage is present at full occlusion, the gain at no occlusion was $V_{\text {diff }}$, or $3.3 \mathrm{~V}$ in this case. With the phototransistor diameter being $0.495 \mathrm{~mm}$, the sensor was assumed to be linear in between $0 \mathrm{~V}$ and $3.3 \mathrm{~V}$, giving a sensor gain of $G_{S}=-\frac{V}{D}=-6667 \mathrm{~V} / \mathrm{m}$.

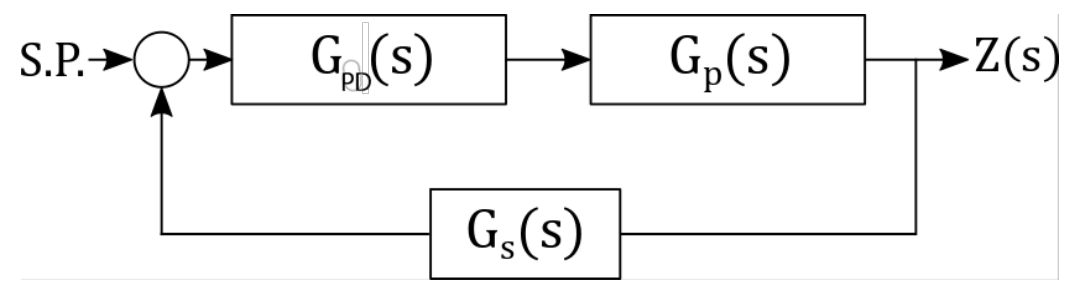

Figure 2, Block Diagram of Simplified System

In Fig. 2, the block diagram of the simplified control system is shown. Letting $\lambda=\frac{C_{i} G_{s}}{M_{s}}$, the closed loop transfer function is given by:

$$
H(S)=\frac{G_{c}(S) G_{p}(S) G_{s}}{1+G_{c}(S) G_{p}(S) G_{s}}=\frac{K_{d} \lambda S+K_{p} \lambda}{S^{2}+K_{d} \lambda S+\left(K_{p} \lambda-\frac{C_{z}}{M_{S}}\right)}
$$

As a second order system, values of $K_{d}$ and $K_{p}$ can be determined to stabilize the system for a desired damping ratio. 
Current control of the electromagnet is not trivial but can be achieved with a proportionalintegral (PI) controller. The electromagnet can be modeled as a resistor with resistance $R_{e}$ and an inductor with inductance $L_{e}$ in series. Parasitic capacitance will be ignored for simplicity. In the Laplace domain, this gives $G_{E}(S)=\frac{I(S)}{V(S)}=\frac{1}{R_{e}+L_{e} S}$. With a PI controller of the form $G_{P I}(S)=$ $K_{p}+\frac{K_{i}}{S}$ to control current in a closed loop system, this simplifies to:

$$
\frac{I(S)}{V(S)}=\frac{\frac{K_{p}}{L_{e}} S+\frac{K_{i}}{L_{e}}}{S^{2}+\frac{\left(R_{e}+K_{p}\right)}{L_{e}} S+\frac{K_{i}}{L_{e}}}
$$

Thus, the full system in block diagram form appears as the following in Fig. 3.

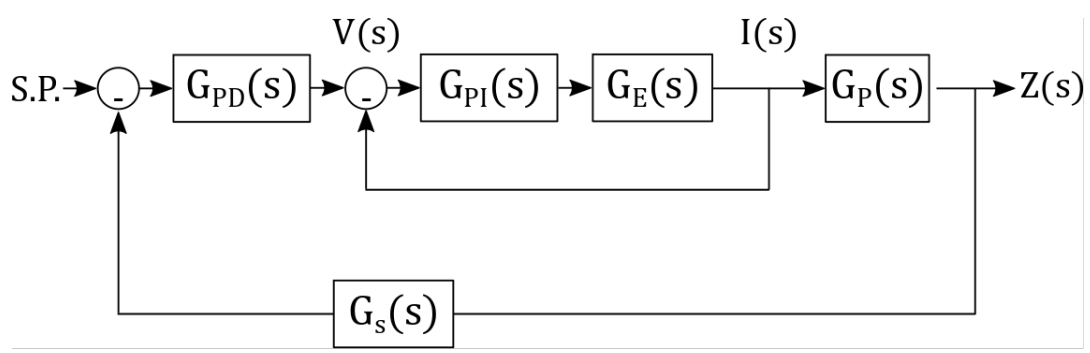

Figure 3, Full Control System Block Diagram

\subsection{Simulation of Theoretical System}

Finite Element Method Magnetics (FEMM) is used to simulate, verify, and determine constants in the theorical system. Although this system is not a truly three-dimensional environment, the problem is axisymmetric around the z-axis, so FEMM can be used.

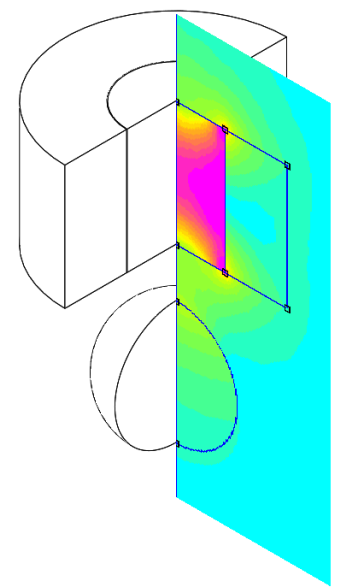

Figure 4, FEMM visualization of $|B|$ around the electromagnet. Purple regions denote high flux density while light blue denotes low density. As stated in (1), the force on the sphere should be proportional to the current through the electromagnet squared. In practice, significant deviations from (1) exist due to magnetic saturation and a variety of other effects. Scripted with MATLAB, FEMM is a powerful tool and is used to determine the parameters to linearize this system. Figure 4 shows a visualization of the magnitude of $B$, the flux density, while the system is at equilibrium.

An equilibrium point $1 \mathrm{~cm}$ from the top of the sphere to the bottom of the electromagnet was arbitrarily chosen because of the relatively low current required to maintain this point. At the same time, this distance retains more than enough space above the sphere for the IR beam.

Although the current controller outputs an analog voltage value in (7), this is infeasible in practice. Instead, pulse-width modulation (PWM) control is used and is the standard in the industry. An H-bridge driven by the current controller can modulate the voltage across the 
electromagnet between $-V_{D},+V_{D}$, and $0 V$. Driven at a high frequency with different duty ratios, any desired $V_{A v g}$ between $-V_{D}$ and $+V_{D}$ can be achieved. The electromagnet, functioning as a powerful inductor, would create massive current and voltage spikes when it is switched on and off so a flyback diode provides a path for inductive current to flow.

A PWM frequency can be chosen to limit the current change in the inductor across one period. This is shown by the back EMF equation, rearranged below:

$$
\frac{d V}{L}=\frac{d i}{d t}
$$

Thus, as $d t$ grows smaller the current rise and drop over one period can be reduced. Once the electromagnet was constructed, it was measured to have an inductance of $41 \mathrm{mH}$. A PWM frequency of $10 \mathrm{Khz}$ was chosen with a drive voltage of 15 volts because the limitation of the controller. These parameters will give a current change across the full PWM period of 0.07 amps. The circuit was modeled in Simulink, and the step response of a modeled inductor connected to an H-bridge is shown in Figure 5 below.

Measuring the current fluctuations across one PWM period in simulation shows a change of only 0.02 amps, which is smaller than expected. As shown in Figure 5, the simulated PI controller with an $\mathrm{H}$ bridge and PWM signal closely follows the theoretical PI response.

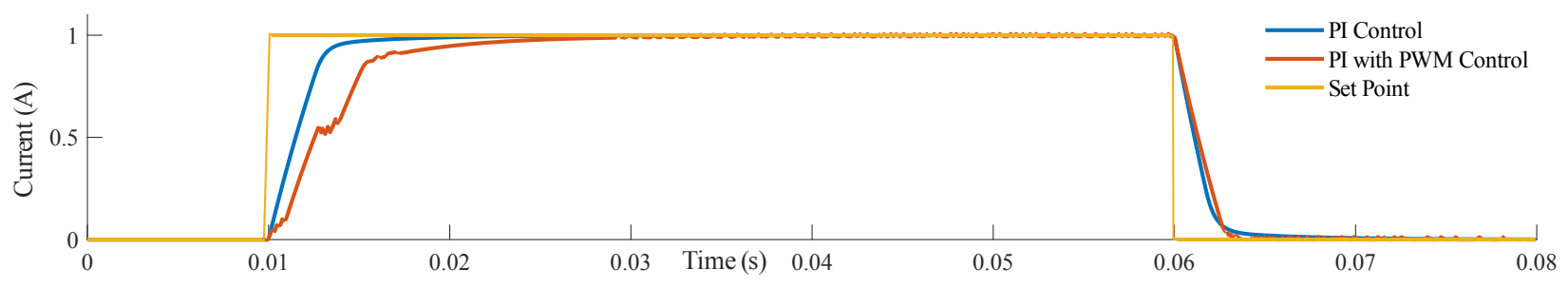

Figure 5, Simulated current controller pulse response

Finally, the PWM coil current simulation can be combined with the description of the sphere dynamics, such as demonstrated in the block diagram in Figure 3. After the constants of the system are found with FEMM and the PD controller is properly tuned, the system is stabilized around $f\left(z_{s}, i_{s}\right)$ as is shown in Figure 6. This simulation should accurately model the final system, with a $10 \mathrm{Khz}$ PWM signal driving a +/-15V H-Bridge configuration.

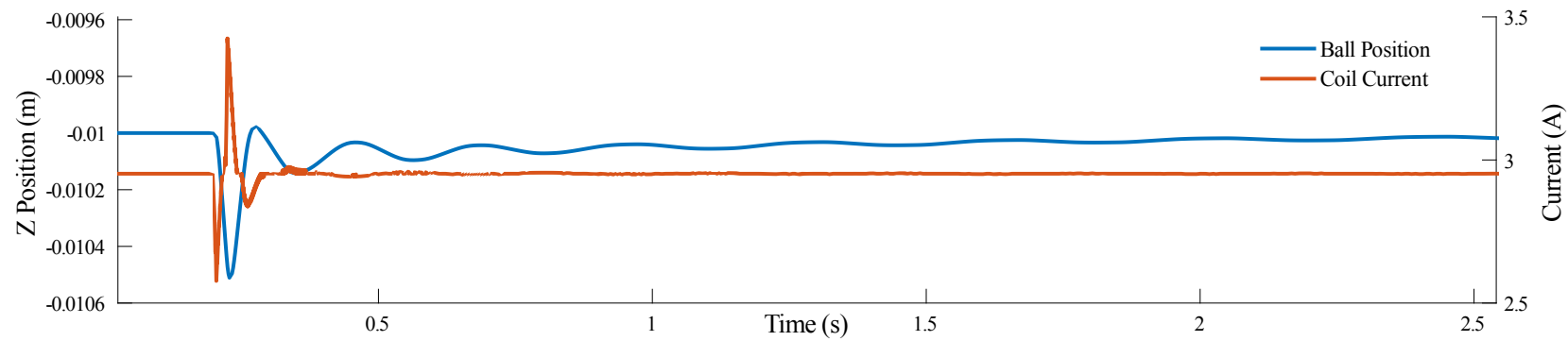

Figure 6, Response of the system with a pulse at $\mathrm{t}=0.2 \mathrm{~s}$ 


\subsection{Hardware Implementation}

A test stand was constructed from two wooden platforms and four threaded screws at the corners. This allowed the height of the electromagnet and the phototransistor beam to be carefully controlled.

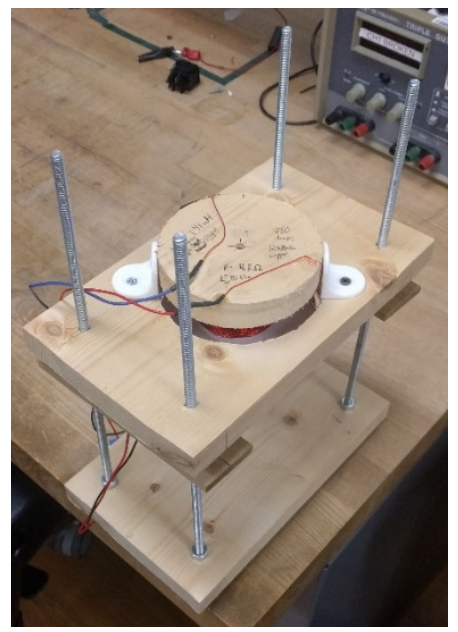

Figure 7, Test stand with electromagnet
The electromagnet was constructed with optimizations in the size utilized from (Hurley \& Wolfle 1997). They included optimizations for the jacket thickness around the electromagnet, but no jacket for the electromagnet was used because of difficulties in manufacturing. FEMM simulation showed that the increase in current required without a jacket was large at $86 \%$. This was initially deemed acceptable. The electromagnet core was lathed to the optimal diameter from a stock steel part. A soft iron core would have been ideal as it has a lower magnetic coercivity and a higher magnetic permeability than steel. Pure iron is hard to obtain, however, and FEMM tests using steel as a core resulted in a $<1 \%$ increase in current.

The gauge of the wire and the turn number in the electromagnet were influenced by several factors. As the electromagnet was being wound by hand, extremely large turn numbers with high gauge wire would be problematic. Low gauge wire would require lower voltage and higher current but would also increase the heat dissipated in the MOSFETs due to lower coil resistance.

A medium gauge wire of 22 AWG was selected. From other papers, a packing efficiency of $\sim 50 \%$ in the coils was estimated, although it was unknown if these electromagnets were mechanically or hand wound (Hurley \& Wolfle, 1997; Wong, 1986). It was found that the optimal coil region could contain an estimated 780 turns. With this gauge wire and turn number, FEMM simulation showed that 2.9 amps were required. The estimated length of the wire needed for the electromagnet was $58 \mathrm{~m}$, which gave a coil resistance of $3 \Omega$ and equilibrium voltage of $8.7 \mathrm{~V}$. All of these were well $(\sim 0.5)$ below the maximum voltage and current ratings of the power MOSFETs first purchased. In practice, hand winding the electromagnet yielded a much lower packing efficiency than estimated. This resulted in a higher coil resistance than predicted at $4.8 \Omega$ and a slightly larger coil for 780 turns. The increased resistance raised the equilibrium voltage to $14 \mathrm{~V}$, which made current control in the electromagnet more challenging.

The first two attempts produced unsuccessful results. The first was made with a basic operational amplifier circuit, modeled off a design described in Taylor (2016). However, electrical issues were hard to debug with only an oscilloscope. Additionally, any modification of the circuit or control parameters required physically replacing parts. This turned out to be time-consuming and wasteful, so this approach was quickly abandoned. Next, a TI Launch-Pad microcontroller was used to control the MOSFETs. The PWM output signals to the MOSFETs worked, but the control systems were challenging to implement. Additionally, significant chatter was found in the electromagnet. This was solved with a stop gap measure of epoxy, but this issue was noted as a potential problem for future electromagnet design. 


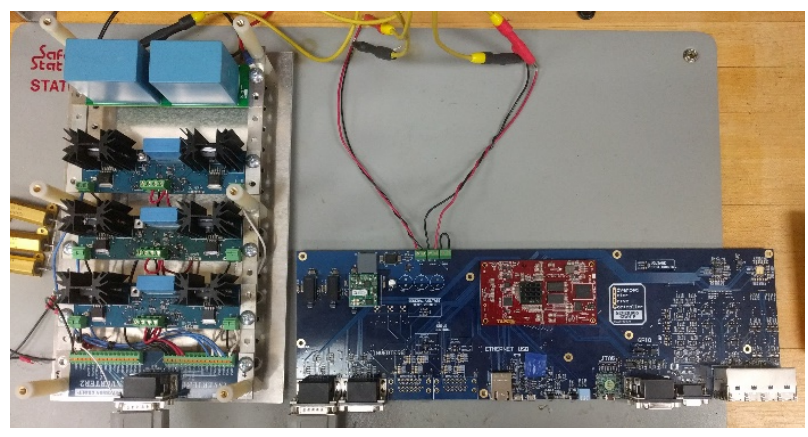

Figure 8, The AMDC connected to three inverter boards

A Zedboard, which has many I/O ports and a Zynq processor was chosen for the next iteration. The Severson lab already had one available, and a Zedboard had previously been used by Prof. Severson to implement a bearingless motor controller. To interface the Zedboard with the circuit, PMod current sensors were used to measure the coil current and a PMod open drain driver was purchased to control the current. However, the output driver barely met the voltage constraints needed at equilibrium. Since the maximum voltage was close to the voltage needed to maintain equilibrium current, this system lacked sufficient bandwidth. A more powerful option for controlling the system became available at this time, the AMDC.

The AMDC being developed by the Severson group has many advantages over the other systems. It is designed as a carrier card for the PicoZed and can drive eight full bridges to produce voltages much higher than needed in this project. Two analog to digital converters provide a total of 16 input channels. The controller, a PicoZed, contains a Zynq-7030 SoC like the one on the Zedboard. The Zynq provides an ARM Cortex-A9 processor and a Kintex-7 fieldprogrammable gate array (FPGA). These are tightly coupled to maximize data throughput between the systems.

This system will be more than powerful enough to provide the control for the one-dimensional demonstration and the full three-dimensional prototype. As it is still under development, a significant amount of time was spent debugging the design and firmware running on it. However, the power and versatility of the AMDC outweighs this initial start up time cost as it will be used through the coming year to continue to develop the full 3D system.

\section{Discussion}

Quite a few lessons were learned over the course of this work, and all of them will be invaluable to continue the construction of the full system into the future. The system has been completely and accurately modeled so that all control parameters have been determined. Once the AMDC is fully operational, the parameters determined in simulation for feedback control will be used on a PI and PD controller running on it.

Several additional improvements to the electromagnet and other aspects of the system are planned. Major inefficiencies exist with the electromagnet that can be solved by constructing a new one. Simply machining and constructing a jacket of iron or steel will reduce the current required by almost $1 / 2$. The issues with chattering in the electromagnet and inefficiency in the winding will be addressed with a new winding machine and epoxied wire. The winding machine can create coils with much more accurate winds than the hand-wound electromagnet used in this experiment. This will increase packing efficiency to levels not achievable with hand winding. 
Specialized magnet wire with a layer of bonding epoxy on the exterior can be used and baked after winding. The epoxy sets after baking, setting the coil and physically constraining the windings. Together, these can increase the efficiency of the electromagnet and bring the forces created closer to the simulated values.

With a fully tested controller, enhanced electromagnet design and a better understanding of how to simulate and stabilize the one-dimensional system, the full prototype will be worked towards through the coming year.

\section{Acknowledgments}

This material is based upon work supported by NASA under Award No. NNX15AJ12H, issued through Wisconsin Space Grant Consortium, and any opinions, findings, and conclusions or recommendations expressed in this material are those of the author(s) and do not necessarily reflect the views of the National Aeronautics and Space Administration.

I would like to thank my mentor, Prof. Eric Severson, for his advice and guidance through many designs and challenges. I would also like to thank the WEMPEC lab manager, Kyle Hanson, and the WEMPEC staff, for their advice, suggestions, and help with troubleshooting.

\section{References}

C.U.Mba, H.A.Gabbar, S.Marchesiellob, A.Fasanab, \& L.Garibaldi. (2017). Fault Diagnosis in Flywheels: Case Study of a Reaction Wheel Dynamic. International Journal of Performability Engineering, 13(4), 362-373.

Carrara, V., \& Kuga, H. K. (2015). Current and Speed Control Operating Modes of a Reaction Wheel. Applied Mechanics and Materials, 706, 170-180.

Hurley, W. G., \& Wolfle, W. H. (1997). Electromagnetic Design of a Magnetic Suspension System. IEEE TRANSACTIONS ON EDUCATION, 40(2), 124-130.

Hurley, W. G., Hynes, M., \& Wölfle, W. H. (2004). PWM Control of a Magnetic Suspension System. IEEE TRANSACTIONS ON EDUCATION, 47(2), 165-173.

Iwakura, A., Tsuda, S.-i., \& Tsuda, Y. (2008). Feasibility Study on Three Dimensional Reaction Wheel. The 57th International Astronautical Congress, (pp. 51-57). Valencia.

Taylor, E. (2016). Magnetic Levitation. Retrieved from Uzzors2k: http://uzzors2k.4hv.org/index.php?page=magneticlevitation

Wong, T. H. (1986). Design of a Magnetic Levitation Control System An Undergraduate Project. IEEE TRANSACTIONS ON EDUCATION, 196-200. 